\section{Conhecimento e uso do Sistema de Informações sobre Orçamentos Públicos em Saúde (SIOPS) pelos gestores municipais, Pernambuco, Brasil}

\author{
Knowledge and use of the Information System on \\ Public Health Budgets (SIOPS) by municipal health \\ administrators, Pernambuco State, Brazil
}

Keila Silene de Brito e Silva 1 Adriana Falangola Benjamin Bezerra 2 Islândia Maria Carvalho de Sousa ${ }^{3}$ Rogério Fabiano Gonçalves ${ }^{2}$

\section{Introdução}

\footnotetext{
${ }^{1}$ Faculdade de Saúde Pública, Universidade de São Paulo, São Paulo, Brasil. 2 Departamento de Medicina Social, Universidade Federal de Pernambuco, Recife, Brasil.

3 Centro de Pesquisas Aggeu Magalhães, Fundação Oswaldo Cruz, Recife, Brasil.

Correspondência

K. S. B. Silva

Faculdade de Saúde Pública Universidade de São Paulo. Rua Itambé 315, apto. 151, São Paulo, SP 01239-001, Brasil.

britokeila@hotmail.com
}

\begin{abstract}
Considering the importance of Brazil's Information System on Public Health Budgets (SIOPS) as a tool for planning, management, and social control of public expenditures in health, this article aimed to evaluate the relationship between the regularity of data entry into the SIOPS and knowledge and use of the system by municipal health administrators in Pernambuco State, Brazil. Ten municipalities were selected from the State's five meso-regions, five of which entered information into the system and five only on an irregular basis. Semi-structured interviews were performed with the municipal health secretaries. Analysis of the data showed that command of information technology and knowledge of the System do not affect the regularity of data entry, as a function of the distance between the Municipal Health Secretariat administrators and the SIOPS, such that the data are normally entered by outsourced services. Thus, the resulting information has not been fully explored by systems administrators as a management tool.
\end{abstract}

Health Evaluation; Information Systems; Health Financing; Health Management
Dos anos 90 aos dias atuais, mudanças importantes ocorreram no financiamento do Sistema Único de Saúde (SUS), tendo algumas destas impactado significativamente no processo de descentralização ao substituir pagamento por produção de serviços pela transferência fundo a fundo, baseada no critério per capita, especialmente no que diz respeito à atenção básica 1 .

De acordo com Sousa et al. 1, o financiamento tem sido o grande impulsionador na implantação de programas/atividades no âmbito de estados e municípios. Apesar disso, o modelo de participação do financiamento federal, geralmente com recursos com destinação pré-definida, ditos "carimbados", força os municípios a uma política vertical, atendendo mais às necessidades de convênios e programas do que à sua realidade epidemiológica.

Com o processo de descentralização é inegável que os municípios tenham assumido grande parte das responsabilidades previstas. Para Arretche 2 , os governos locais tornaram-se essenciais na provisão de serviços de saúde e hoje ocupam um importante papel no seu financiamento.

O crescente compartilhamento do financiamento do SUS e o avanço na regulamentação das transferências demandaram a criação de um sistema de informação que possibilitasse a coleta, o processamento e a divulgação de dados sobre a receita e despesa com saúde nos três níveis de 
gestão ${ }^{3}$. Uma primeira iniciativa nesse sentido foi implementada pelo Conselho Nacional de Saúde, em 1993, com o propósito de obter informações sobre despesas em saúde.

No ano de 1999, o Ministério da Saúde implantou o Sistema de Informações sobre Orçamentos Públicos em Saúde (SIOPS) ${ }^{4}$, caracterizado como um instrumento fundamental para o acompanhamento da receita e despesa em saúde, permitindo analisar, por exemplo, o percentual de recursos próprios aplicados em saúde, de acordo com a Emenda Constitucional $n^{o}$. 29 (EC29); a receita de impostos e transferências constitucionais e legais, total e por habitante; as transferências do Ministério da Saúde totais e por habitante; o detalhamento das despesas com ações e serviços de saúde como de pessoal, serviços de terceiros e medicamentos, entre outros 5 .

Os dados gerados pelo SIOPS constituem também insumos para o planejamento e gestão, para a elaboração e implementação dos Planos Diretores de Regionalização (PDRs) e dos Planos Diretores de Investimentos (PDIs), uma vez que há previsão de aportes, pelo conjunto dos governos, para a construção de uma rede regionalizada e hierarquizada de serviços de saúde, na busca da melhoria da acessibilidade e da integralidade da atenção à saúde. Acresce ainda que a Portaria GM/MS no. 2.047/026 6 e a Resolução CNS $n^{o}$. 322/037 7, que dispõem sobre as Diretrizes Operacionais para a Aplicação da EC29, de 2000, adotam o SIOPS como referência para o acompanhamento, a fiscalização e o controle da aplicação dos recursos vinculados em ações e serviços públicos de saúde.

No Estado de Pernambuco, segundo Sousa et al. 1, 40\% dos municípios no primeiro semestre de 2004 não transmitiram informações para o SIOPS. Considerando que as informações prestadas ao sistema são declaratórias e que, no caso dos municípios, não estão sujeitas à verificação da consistência, os $60 \%$ que transmitem a informação podem estar sujeitos a diferentes graus de precisão nos dados disponibilizados. Bezerra 8 , ressalta que essa constatação evidencia a "variação e irregularidade na alimentação do SIOPS e, sobretudo, fragiliza a utilização do sistema como ferramenta de informação gerencial".

Um estudo recente 8 , desenvolvido nos municípios de Pernambuco para os anos de 2000 a 2006, identificou um percentual de $32,9 \%$ de pendências na alimentação do SIOPS no ano de 2006 e um percentual de apenas 3,79\% de cumprimento do prazo de envio dos dados ao SIOPS, caracterizando uma irregularidade na transmissão das informações e um atraso significativo na alimentação do sistema. Sendo o conhecimento construído a partir da acumulação de infor- mações consolidadas por meio da atualização permanente e do confronto de antigas e novas informações 9 , o atraso na alimentação interfere diretamente no processo de construção do conhecimento, enfraquecendo o SIOPS como recurso político e dificultando sua utilização para fins de controle social.

Sistemas de informação como o SIA (ambulatorial) e o SIH (hospitalar) foram criados, essencialmente, para acompanhar a produção de pagamento de procedimentos e vêm sendo estudados e aperfeiçoados ao longo dos anos 10. O SIOPS, por sua vez, foi criado, essencialmente, para ser uma ferramenta de monitoramento, fundamental para o exercício da regulação e avaliação financeira da contrapartida municipal.

As dificuldades para alimentação e utilização do SIOPS impossibilitam ao gestor do Fundo e ao Conselho de Saúde analisar as informações sobre o comportamento das receitas e das despesas em diferentes níveis de detalhamento. Obstaculizam, também, a comparação dos dados de estados e municípios segundo critérios regionais ou de porte populacional, e a consolidação de dados sobre o gasto público em diferentes formas de agregação.

Considerando a importância do SIOPS como ferramenta para o planejamento, gestão e controle social dos gastos públicos em saúde, este artigo teve como objetivo avaliar o conhecimento e a utilização do SIOPS pelos gestores municipais de Pernambuco e sua relação com a regularidade na alimentação do sistema, sendo norteado pelas seguintes questões de pesquisa 11: os gestores de saúde municipais conhecem e utilizam o SIOPS? Conhecer e utilizá-lo reflete em uma maior regularidade na sua alimentação?

\section{Procedimentos metodológicos}

O estudo foi desenvolvido pelo grupo de pesquisa em Economia Política da Saúde, da Universidade Federal de Pernambuco (UFPE), sendo parte do projeto Avaliação do SIOPS e Capacitação de Gestores Municipais para a Atualização e Qualificação dos Dados no Uso da Tecnologia da Informação, iniciado em janeiro de 2006 e financiado pelo Conselho Nacional de Desenvolvimento Científico e Tecnológico (CNPq).

Por se tratar de um estudo que "examinou por meio de um procedimento científico as relações que existem entre os diferentes componentes de uma intervenção" 12 (p. 31), no caso, o conhecimento, o uso e a regularidade na alimentação do SIOPS, o mesmo se caracteriza como uma pesquisa avaliativa (Figura 1). 
Componentes de intervenção na avaliação da regularidade na alimentação do Sistema de Informações sobre Orçamentos Públicos em Saúde (SIOPS).

SIOPS

Sistema de informação que apura os gastos em saúde realizados pelas três esferas de governo

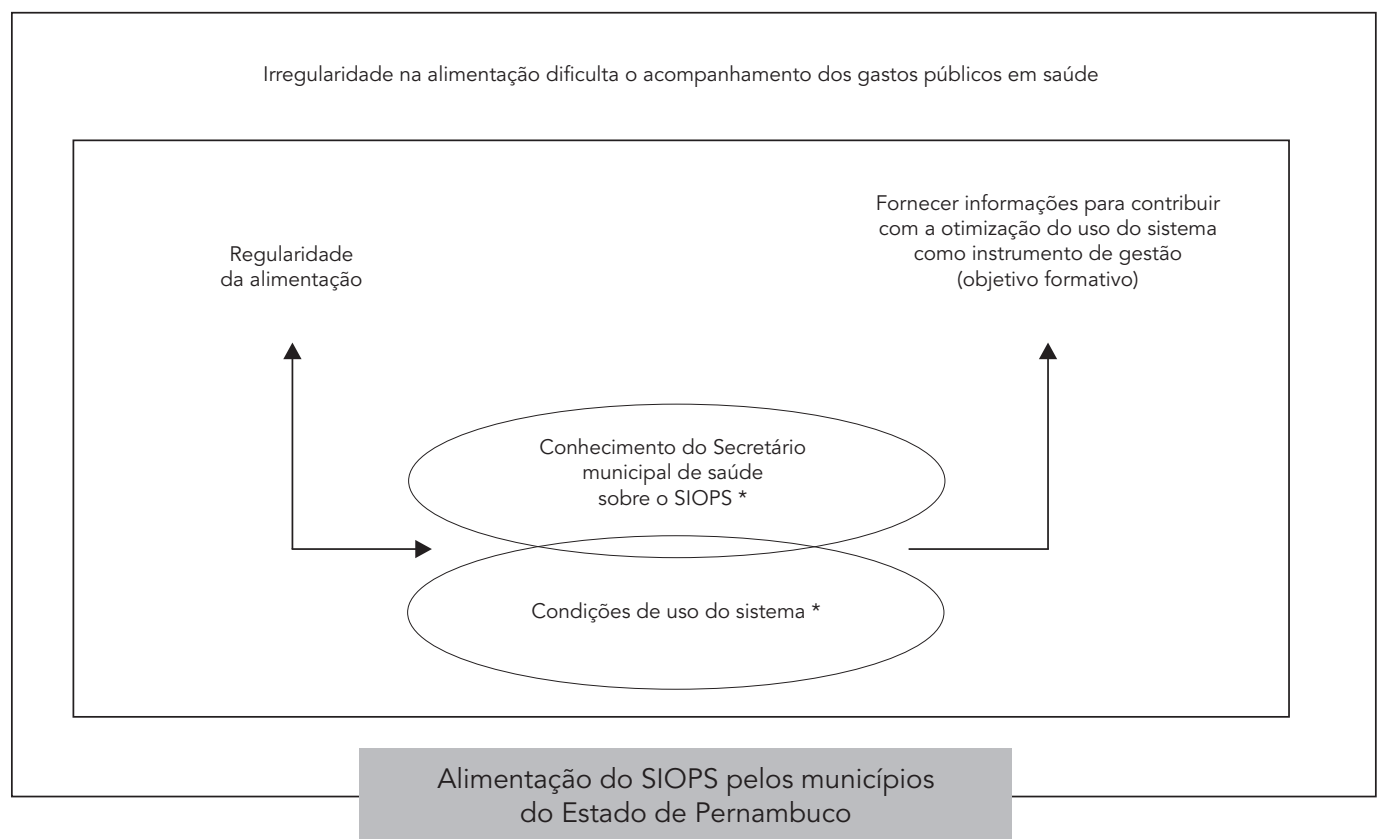

* Componentes da intervenção.

Fonte: elaboração própria com base na figura de componentes de intervenção de Contandriopoulos et al. 12

Em investigações que envolvem sistemas computadorizados e as percepções e comportamento dos usuários acerca dos mesmos, há a necessidade de explorar diversos métodos de pesquisa, sobretudo os qualitativos 13 . Nesse sentido, foi elaborado um estudo de natureza qualitativa, envolvendo dez Secretarias Municipais de Saúde (SMS), distribuídas nas cinco mesorregiões do estado, na perspectiva de responder às questões norteadoras da pesquisa com base nos sentidos que os secretários municipais de saúde de Pernambuco atribuem à sua experiência 14 com SIOPS.

Para seleção dos secretários foi realizado o levantamento dos municípios regulares e irregulares na alimentação do SIOPS em cada mesorregião do estado. Para essa classificação foram analisados os relatórios semestrais e anuais dos municípios na base de dados do sistema, considerando o período de 2000 a 2006.
Como destacado anteriormente, este trabalho é parte de uma pesquisa-mãe 8 que abrangeu todo o estado, desenvolvendo estudos quantitativos e qualitativos acerca do SIOPS e utilizando diferentes variáveis adequadas a cada objeto de estudo. A opção por restringir o presente estudo à variável regularidade foi motivada pelo destaque que a mesma obteve entre as fragilidades mais evidenciadas nas pesquisas anteriores desenvolvidas pelo grupo de pesquisa sobre o SIOPS no Estado de Pernambuco.

Os critérios para classificação da regularidade na alimentação do sistema foram baseados nos parâmetros estabelecidos no estudo de Bezerra 8 intitulado: Avaliação da regularidade na alimentação do SIOPS pelos municípios de Pernambuco. Nesse sentido, as variáveis consideradas para a avaliação da regularidade compreenderam a quantificação dos municípios adimplentes e inadimplentes com a alimentação do sistema e 
o cumprimento do prazo para envio dos dados ao SIOPS.

O critério de regularidade estabelecido obedeceu ao que prevê o art. 52 da Lei de Responsabilidade Fiscal (LRF) 15 e do Anexo de Metas Fiscais do Relatório Resumido da Execução Orçamentária (RREO), que determinam aos estados e municípios o prazo de 30 dias, após o encerramento de cada semestre, para a publicação do RREO por meio dos formulários do SIOPS.

Após o levantamento da adimplência dos municípios quanto à alimentação do SIOPS, foram selecionados dois de cada mesorregião, sendo um regular e outro irregular, com secretários de saúde ocupando o cargo por, no mínimo, um ano, visto que um período inferior a este poderia dificultar a obtenção de informações pela pouca propriedade do entrevistado acerca do tema.

Para a coleta dos dados foi utilizada uma entrevista semi-estruturada que abordou questões referentes ao perfil dos SMS, às condições de uso da Internet na prefeitura/secretaria e ao conhecimento/utilização do SIOPS, além de aspectos que podem ter influenciado na alimentação regular ou irregular do Sistema. O tipo de entrevista escolhido se justificou por se tratar de um instrumento que apesar de utilizar um roteiro prévio para o direcionamento das perguntas, não impede que os entrevistados falem livremente sobre o assunto abordado 16. O registro das informações foi realizado por meio de gravações de áudio e anotações de aspectos relevantes durante e após as entrevistas.

Os dados foram analisados por meio da análise de conteúdo, um procedimento clássico empregado para trabalhar material textual ${ }^{17}$. Como ferramenta foi utilizado o NVivo 2.0 (QRS International Pty, Doncaster, Austrália), um programa especificamente projetado para auxiliar na análise qualitativa, que facilitou o processo de categorização dos dados 18,19. Posteriormente, procedeu-se o cruzamento das informações para verificar se a regularidade na alimentação estava relacionada ao conhecimento e uso do SIOPS pelos secretários.

Para garantir o direito de anonimidade, os entrevistados foram identificados por nomes fictícios, sendo utilizadas palavras do cotidiano da gestão em saúde.

\section{Resultados e discussão}

Na perspectiva de favorecer uma visualização mais abrangente dos dados, foram elaborados dois diagramas, sendo o primeiro referente à relação do conhecimento e uso do SIOPS com a regularidade na alimentação (Figura 2), e o segundo as justificativas dos gestores entrevistados pela regularidade ou irregularidade na alimentação do SIOPS (Figura 3).

\section{O perfil dos atores entrevistados}

Entre os dez secretários entrevistados, nove tinham nível superior completo na área da saúde, sendo cinco destes com pós-graduação no campo da saúde coletiva. Sete secretários ocupavam o cargo há mais de um ano, e três o exerciam há exatamente um ano. Em relação à experiência anterior em cargo/função pública, apenas três não exerceram.
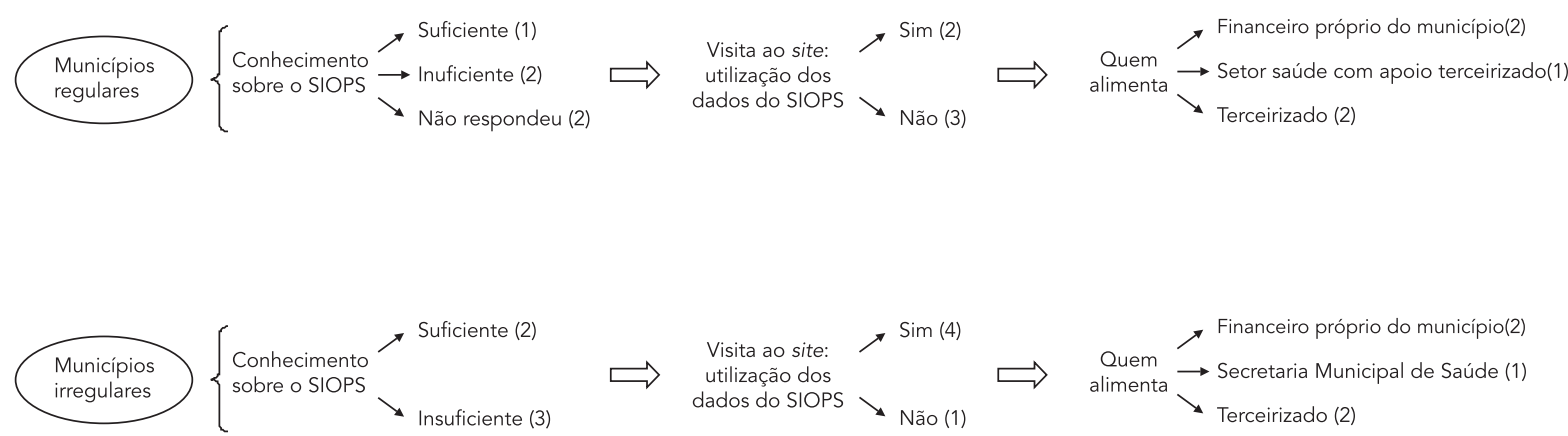
Justificativas dos gestores à regularidade ou irregularidade na alimentação do Sistema de Informações sobre Orçamentos Públicos em Saúde (SIOPS).

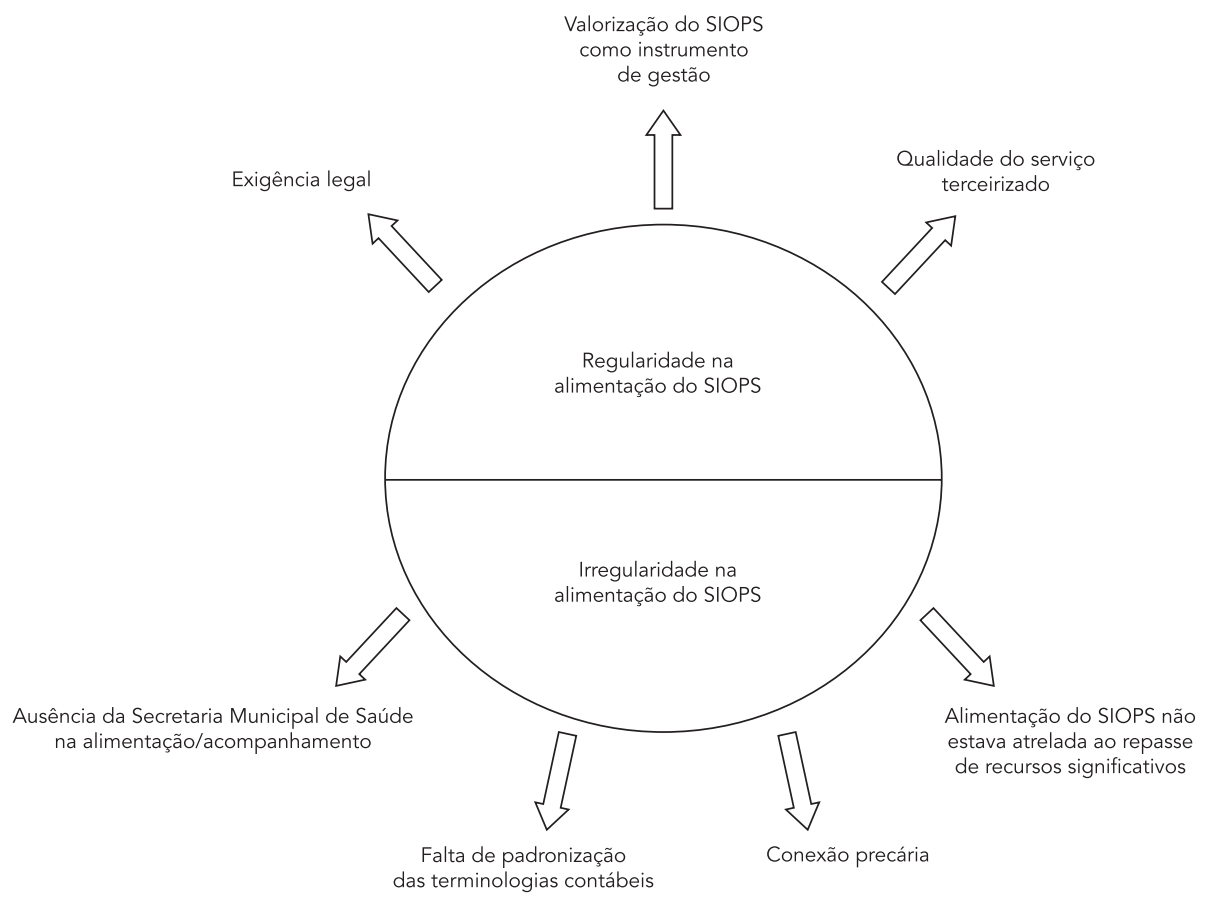

\section{O uso da informática pelos atores}

Em relação ao uso da informática, seis referiram ter um domínio suficiente e sete a utilizavam tanto para a Gestão do Sistema de Saúde quanto para uso pessoal. $\mathrm{O}$ acesso à Internet estava presente em todas as prefeituras, entretanto, a conexão foi considerada insatisfatória em três municípios.

A conexão precária, referida pelos entrevistados, demonstrou ser um fator que pode influenciar na irregularidade da alimentação de alguns municípios, como destacou este entrevistado: “a gente tem dificuldade na transferência dos dados porque lá a gente não tem banda larga, então a gente tem muito problema de mandar via Internet. Cai muito a conexão" (Planejamento).

A diversidade acerca da conexão (precária ou satisfatória) caracteriza a heterogeneidade entre as secretarias municipais no que diz respeito à infra-estrutura de informática. De acordo com Branco 9, a maioria dos municípios brasileiros efetua apenas procedimentos de coleta de dados para o sistema de âmbito federal ou estadual, enquanto que em outro extremo há secretarias que além desta função, produzem, processam, organizam e analisam a informação em saúde de seu município.

A redução dos municípios a meros coletores de dados comprometeu sua atuação tanto do ponto de vista financeiro quanto técnico, refletindo no atrofiamento da administração municipal acerca dos sistemas de informações e em outras dimensões de sua capacidade técnica, fundamentais no processo de planejamento da saúde 9,20,21.

Observa-se, entretanto, que o domínio de informática não influenciou na regularidade da alimentação, assim como o conhecimento sobre o SIOPS: apenas três entrevistados demonstraram um conhecimento mais consistente sobre o Sistema, os demais apresentaram uma compreensão superficial ou não responderam ao questionamento. Pode-se atribuir esse resultado ao fato de que, na maioria (oito) dos municípios, a alimentação não envolve a gestão de saúde municipal, sendo executada, principalmente, por serviços terceirizados ou secretaria de finanças, como relataram alguns entrevistados: 
"Eu não conheço a pessoa que alimenta o SIOPS. Não sei quem é. É uma assessoria que está ligada à Secretaria de Finanças e não à Secretaria de Saúde. Eu não participo da alimentação" (Organização).

"A gente nem se preocupa, acha que eles [serviço terceirizado] fazem tudo e a gente até confia demais, mas sempre a gente se despreocupa quando tem gente especializada naquela área" (Programação).

A ausência da gestão de saúde municipal na alimentação do SIOPS e o repasse da responsabilidade aos serviços terceirizados, como observado no estudo em questão, podem ser justificados pela falta ou deficiência de recursos humanos qualificados para análise das informações obtidas nas secretarias ?.

A terceirização associada a serviços de administração pública apesar de não se constituir uma prática nova e estar assegurada legalmente, tem sido cada vez mais criticada. De acordo com Campos 22, os parcos recursos que a administração pública dispunha, inclusive para tentar acompanhar as novas tecnologias, foram o grande impulsionador do investimento na terceirização. Ver-se impotente e ao mesmo tempo constatar que não seria necessário despender tantos recursos financeiros, material e humano para gerir um número elevado de servidores no exercício de atividades meio, causando entraves cada vez maiores no andamento dos serviços e sobrecarregando a folha de pagamento, levaram o gestor a centrar-se nas atividades fim, abrindo espaço para a implantação da terceirização de alguns serviços.

A contratação do serviço terceirizado na perspectiva de reduzir custos pode surtir efeito contrário à administração pública; enquanto as empresas privadas, por perseguirem o lucro, investem em treinamento para o seu pessoal efetivo que executa as atividades finalísticas, e ao contratarem terceiros exigem que estes trabalhadores já venham com treinamento específico à custa da prestadora, nos serviços públicos ocorre o inverso 23 .

Segundo Alves 23, a terceirização na administração pública prejudica, diretamente, uma das maiores conquistas da sociedade: a democracia, visto que a fragilidade de vínculo restringe as reivindicações dos funcionários. $\mathrm{O}$ autor destaca ainda que o envolvimento da verba pública com o setor privado e a transferência de responsabilidade para uma empresa particular, além de tornar o Estado dependente do setor privado, os efeitos oriundos dos serviços terceirizados afetam uma grande quantidade de contribuintes.

A presença marcante do serviço terceirizado na alimentação do SIOPS torna-se também preocupante pelo distanciamento gerado entre o sistema e a gestão de saúde municipal, principal responsável pelas informações de seu município. O setor de finanças também tem assumido a alimentação em algumas localidades sem envolver a secretaria de saúde neste processo, evidenciando ainda mais o hiato existente entre as SMS e o Sistema.

\section{O SIOPS e a gestão municipal}

Os entrevistados consideraram o SIOPS uma importante ferramenta para o acompanhamento e direcionamento da gestão, entretanto, a maioria (oito) nunca utilizou os seus dados e quatro secretários afirmaram nunca ter visitado a página. Com relação aos que afirmaram ter utilizado os dados, apenas um o fez como instrumento de avaliação do município, que corresponde, exatamente, ao gestor que atribuiu a regularidade na alimentação dos dados à "valorização do SIOPS como instrumento de Gestão”, podendo ser visualizado no trecho de fala a seguir:

"Uma das questões fundamentais, se você quiser fazer, de fato, uma gestão minimamente participativa, com qualidade, é você ter uma base de informação boa. Então a gente investiu nisso na medida do possível, partindo desse princípio de que se o município ele quer investir ele tem que se conhecer bem e isso nos ajudou, de fato, a fazer um planejamento, minimamente, regular" (Participação).

O relato de um dos entrevistados demonstrou, inclusive, o desconhecimento da utilidade das informações do SIOPS para a gestão municipal:

"Os municípios não se beneficiam com o retorno dessas informações, não há uma retroalimentação. Essas informações prestadas não são revertidas para contribuírem com o planejamento das ações municipais. Não sei para que servem essas informações. Pode ser que sirvam para engordar as estatísticas do governo. Deveriam contribuir com os municipios" (Comunicação).

Respostas superficiais sobre o que seria importante aprender sobre o SIOPS, reforçaram a baixa propriedade acerca do tema pelos entrevistados. A ausência da SMS na alimentação foi também enfatizada por alguns gestores como justificativa pela alimentação irregular:

“Eu próprio recebo o relatório do SIOPS quando ele tá pronto! Feito! Quer dizer, a Secretaria de Saúde não participa da alimentação desses dados. E eu suponho, tenho certeza que muitas coisas ficam de fora, tá certo? (...) Eu acredito que o município está nessa dificuldade [irregularidade na alimentação] porque o corpo técnico, o secretário e o departamento de planejamento financeiro 
da Secretaria de Saúde não estão participando, não está sendo consultado para a alimentação do SIOPS (...). Em minha opinião deveria ser diferente. Acho que nós da Secretaria de Saúde é que deveríamos ser treinados e capacitados para alimentar o SIOPS, porque nós é que somos os detentores da informação verdadeira" (Organização).

Em relação à participação em cursos de capacitação para utilização do SIOPS, apenas dois secretários foram submetidos a capacitações referentes ao tema, destes, apenas um referiu se considerar capacitado.

A qualidade da informação está intrinsecamente vinculada à sua utilização na gestão do Sistema de Saúde 24. A manipulação regular dos dados possibilita o aprimoramento dos sistemas de informação: quanto mais utilizado maior a possibilidade de identificar as falhas no banco de dados 25,26. Nesse sentido, a não utilização do SIOPS como instrumento de gestão retrata a insuficiente intimidade entre os gestores e o SIOPS, e faz da sua alimentação um mero "cumprimento de tabela", comprometendo a qualidade da informação, o processo decisório e, conseqüentemente, os resultados a serem obtidos 9 .

Outro fator que pode contribuir para esse quadro é a cultura do não avaliar/acompanhar. De acordo com pesquisa realizada pelo Ministério da Saúde 27, o Plano de Saúde, por exemplo, é um dos instrumentos mais citados para utilização no processo de planejamento entre os municípios brasileiros, entretanto não são avaliados pela maioria das secretarias. Nas regiões Norte, Nordeste e Centro-oeste, a proporção de secretarias que realizam a avaliação é ainda menor que nas regiões Sul e Sudeste.

Diante do cenário apresentado, o SIOPS fica reduzido a uma ferramenta de coleta de dados estatísticos para o controle dos gastos por parte dos órgãos de fiscalização, como ocorria antes da sua estruturação com as planilhas eletrônicas criadas pelo Departamento de Informática do SUS (DATASUS) para apurar o perfil do financiamento e do gasto em saúde, e perde a sua potencialidade de instrumentalizar a gestão na tomada de decisão e de subsidiar as reivindicações sociais, enfraquecendo o exercício da cidadania 20. Essa situação torna-se ainda mais preocupante quando se trata de um Sistema que reúne informações sobre financiamento e gasto com saúde pública 3 .

A fragilidade dos gestores no uso do SIOPS é também conseqüência do investimento insuficiente em cursos de capacitação, como pôde ser observado nos resultados do presente estudo. Por mais recursos de "ajuda" que existam na página do SIOPS para esclarecimento aos usuários acerca do preenchimento da declaração 3 , a estruturação de cursos práticos para conhecimento e utilização do Sistema é fundamental para instrumentalizar os gestores no uso da ferramenta.

A exigência legal para alimentação do SIOPS é outro aspecto atribuído à regularidade na alimentação:

"Em primeiro lugar, o compromisso da gestão em está alimentado... A gente sempre tem o compromisso de está sempre alimentando todos os sistemas e o fato, também que... meio que o brasileiro gosta assim, cumpre muito quando é cobrado. E o fato de que você não alimentando você fica com pendências, onde você pode ter dificuldades no repasse de novos recursos, isso faz com que você se mantenha sempre em alerta para estar com esse sistema em dia" (Pactuação).

"O prefeito cobra e há conscientização da secretaria com os prazos" (Comunicação).

A atribuição à exigência legal como determinante da regularidade na alimentação do SIOPS, diz respeito ao estabelecimento do Sistema como fonte para comprovação de alocação dos recursos da saúde atendendo à LRF 15. Outro instrumento legal que exige a alimentação regular do SIOPS, corresponde à Portaria no ${ }^{\circ}$ 91/GM de 10 de janeiro de 2007 28, que estabelece no Relatório de Indicadores de Monitoramento e Avaliação do Pacto pela Saúde o indicador referente à proporção da receita própria aplicada à saúde conforme previsto na EC29, tendo como fonte de informação o SIOPS.

Outro aspecto utilizado como justificativa pelos gestores foi o "não atrelamento da alimentação do SIOPS ao repasse de recursos significativos": " muita gente ainda não dá importância ao SIOPS porque ele até algum tempo não limitava o repasse de muita coisa. Muitos gestores levam em consideração isso: se não me traz problema eu vou empurrando com a barriga. Isso vem modificando um pouco, porque o SIOPS hoje ele gera uma situação de inadimplência que impede o repasse de recursos" (Negociação).

$\mathrm{O}$ discurso do gestor acima demonstra que a decisão pela alimentação dos sistemas de informação está restrita às penalidades envolvidas no seu descumprimento. Dessa forma se observa, mais uma vez, o quanto a compreensão desses sistemas enquanto ferramenta para a gestão está limitada.

Segundo Cohn \& Westphal 29 (p. 120), “ $a$ maneira de implantação do SUS parece conformar uma determinada prática de coleta e sistematização das informações, por parte dos municípios independente do seu porte" que são motivadas, sobretudo, para as questões de faturamento e financiamento dos serviços, sendo restrito o uso 
das informações para proposições inovadoras no nível municipal.

A falta de padronização das terminologias contábeis é também referida como responsável pelo atraso na alimentação do sistema: " a gente teve alguns problemas em 2006, porque assim, em 2005 a gente pegou muitos restos a pagar e tinha uma confusão muito grande dos contadores do que é que se considera restos a pagar. OSIOPS considera restos a pagar de uma forma e o Tribunal de Contas do Estado considera outra. Então isso gerou muita confusão quando eu assumi a secretaria. O que é que se entende por despesa liquidada e despesa paga? O SIOPS considera de uma forma e o Tribunal de Contas considera outra em relação à prestação de contas" (Gestão).

Questões referentes à problemática da não padronização de termos contábeis têm sido alvo de discussão em vários fóruns no país. No Seminário de Pesquisa em Financiamento da Saúde, realizado em São Paulo, que reuniu estudiosos da temática do Siops de vários estados, foi registrada a necessidade de padronização das terminologias, na perspectiva de reduzir as dificuldades tanto para a realização de estudos comparativos quanto na realização de atividades de fiscalização e acompanhamento dos órgãos competentes (Tribunal de Contas, Departamento Nacional de Auditoria do SUS, Conselhos de Saúde), em função de não haver uma uniformização nos termos adotados pelos entes federados na realização dos balancetes contábeis.

De acordo com o Tribunal de Contas do Estado (TCE), a Secretaria do Tesouro Nacional (STN) tem trabalhado na perspectiva da padronização dos termos contábeis, com previsão de início das novas regras em 2009.

\section{Considerações finais}

A utilização da abordagem qualitativa no estudo do sistema de informação permitiu compreender a relação estabelecida entre o SIOPS e o usuário gestor, sendo identificada uma relação inexistente ou de exclusivo cumprimento a fim de atender a uma demanda legal.

O estudo constatou que o conhecimento e uso do SIOPS pelos gestores municipais do Estado de Pernambuco não influenciam diretamente na regularidade da alimentação do sistema, tendo em vista a baixa participação das secretarias de saúde neste processo. Em contrapartida, foram evidenciados outros aspectos que têm interferido tanto na regularidade da alimentação quanto na utilização do sistema como instrumento de gestão.
A atribuição da atividade de alimentação do SIOPS, principalmente, a serviços terceirizados, pode estar contribuindo para a participação in suficiente da gestão de saúde municipal na alimentação, bem como a pouca propriedade e interesse dos gestores acerca do tema. Muitas vezes essa participação dos serviços terceirizados não se restringe apenas ao SIOPS, mas historicamente a outros sistemas como o SIH, o que expressa uma utilização limitada da informação.

As informações geradas pelo SIOPS não têm sido exploradas potencialmente pelos gestores enquanto instrumento de gestão. Um maior investimento no fortalecimento das habilidades e competências dos gestores e dos técnicos em saúde para o uso das possibilidades ofertadas pelo Sistema é fundamental para otimizar o uso do SIOPS a favor do Sistema de Saúde.

O SIOPS se diferencia dos demais sistemas de informações porque não está relacionado diretamente às atividades fim, tanto de produtividade como de informação epidemiológica, ele produz essencialmente informação financeira para a gestão e controle social. Desse modo, os estudos que revelam a relação dos gestores com o mesmo podem permitir uma aproximação sobre o gerenciamento financeiro da saúde no lócus de investigação.

Considerando que esta pesquisa fez uso exclusivo da regularidade como variável, tendo em vista o seu objeto de estudo, destaca-se a importância de serem desenvolvidos outros estudos qualitativos acerca do tema que considerem outras variáveis no seu recorte, permitindo a compreensão de diferentes aspectos também relevantes neste contexto. 


\section{Resumo}

Considerando a importância do Sistema de Informações sobre Orçamentos Públicos em Saúde (SIOPS) como ferramenta para o planejamento, gestão e controle social dos gastos públicos em saúde, este trabalho teve como objetivo avaliar a relação entre a regularidade na alimentação do SIOPS e o conhecimento e uso do Sistema pelos gestores municipais do Estado de Pernambuco, Brasil. Foram selecionados dez municípios distribuídos nas cinco mesorregiões do estado, sendo cinco regulares e cinco irregulares na alimentação do Sistema, e aplicada uma entrevista semi-estruturada entre os secretários de saúde dos respectivos municípios. Com base na análise dos dados, foi identificado que o domínio de informática e o conhecimento do Sistema não interferem na regularidade da alimentação, em função do distanciamento entre os gestores das Secretarias de Saúde e o SIOPS, em geral alimentado por serviços terceirizados. Constata-se que as informações geradas não têm sido exploradas potencialmente pelos gestores enquanto instrumento de gestão.

Avaliação em Saúde; Sistema de Informação; Financiamento da Saúde; Gestão em Saúde

\section{Colaboradores}

K. S. B. Silva participou da coleta e análise dos dados e redação do artigo. A. F. B. Bezerra, I. M. C. Sousa e R. F. Gonçalves contribuíram com a coleta dos dados e revisão do artigo.

\section{Referências}

1. Sousa IMC, Silva ASS, Canto A. Financiamento no SUS: a Emenda Constitucional 29 e o Sistema de Informações sobre Orçamentos Públicos em Saúde em Pernambuco. Recife: Núcleo de Estudos em Saúde Coletiva, Centro de Pesquisas Aggeu Magalhães, Fundação Oswaldo Cruz; 2005.

2. Arretche M. Financiamento federal e gestão local de políticas sociais: o difícil equilíbrio entre regulação, responsabilidade e autonomia. Ciênc Saúde Coletiva 2003; 8:331-45.

3. Teixeira HV, Teixeira MG. Financiamento da saúde pública no Brasil: a experiência do Siops. Ciênc Saúde Coletiva 2003; 8:379-91.

4. Ministério da Saúde. A implantação da EC 29: apresentação dos dados do Siops, 2000 a 2003. http:// www.saude.gov.br/ (acessado em 15/Jul/2007).

5. Ministério da Saúde. Emenda Constitucional 29/2000. http://www.saude.gov.br/conasss/emen da (acessado em 07/Dez/2006).
6. Ministério da Saúde. Portaria GM/MS nº. 2047/026. Diário Oficial da União 2002; 7 nov.

7. Conselho Nacional de Saúde. Resolução $\mathrm{n}^{\mathrm{o}}$. 322/037. Diário Oficial da União 2003; 5 jun.

8. Bezerra AFB. Avaliação do SIOPS e capacitação de gestores municipais para a atualização e qualificação dos dados no uso da tecnologia da informação. Relatório de Pesquisa financiada pelo Conselho Nacional de Desenvolvimento Científico e Tecnológico (CNPq). Pesquisa Saúde - DECIT/ SCTIE/MS; 2008. http://pesquisasaude.saude.gov. br/bdgdecit/index.php?lg=BR (acessado em 08/ Out/2008).

9. Branco MAF. Informação em saúde. Rio de Janeiro: Editora Fiocruz; 2001.

10. Bittencourt SA, Camacho LAB, Leal MC. O Sistema de Informação Hospitalar e sua aplicação na saúde coletiva. Cad Saúde Pública 2006; 22:19-30. 
11. Creswell JW. Projeto de pesquisa: métodos qualitativo, quantitativo e misto. Porto Alegre: Editora Artmed; 2007.

12. Contandriopoulos AP, Champagne F, Denis JL, Pineault R. A avaliação na área da saúde: conceitos e métodos. In: Hartz ZMA, organizadora. Avaliação em saúde: dos modelos conceituais à prática na análise da implantação de programas. Rio de Janeiro: Editora Fiocruz; 1997. p. 29-47.

13. Pozzebon M, Freitas HMR. Pela aplicabilidade com um maior rigor científico dos estudos de caso em sistemas de informação. Revista de Administração Contemporânea 1998; 2:143-70.

14. Pope C, Mays N. Métodos qualitativos na pesquisa em saúde. In: Pope C, Mays N, organizadores. Pesquisa qualitativa na atenção à saúde. Porto Alegre: Editora Artmed, 2005. p. 11-9.

15. Brasil. Lei complementar ${ }^{\circ}$. 101, de 4 de maio de 2000. Estabelece normas de finanças públicas voltadas para a responsabilidade na gestão fiscal e dá outras providências. Diário Oficial da União 2000; 5 mai.

16. Martins GA. Estudo de caso: uma estratégia de pesquisa. São Paulo: Editora Atlas; 2006.

17. Flick U. Uma introdução à pesquisa qualitativa. Porto Alegre: Bookman; 2004.

18 Di Gregorio S. Using Nvivo for your literature review. http://www.sdgassociates.com/downloads/ literature_review.pdf (acessado em 02/Jan/2007).

19. Guizzo BS, Krziminski CO, Oliveira DLLC. O software QSR NVIVO 2.0 na análise qualitativa de dados: ferramenta para a pesquisa em ciências humanas e da saúde. Rev Gaúch Enferm 2003; 24: 53-60.
20. Branco MAF. Informação em saúde como elemento estratégico para a gestão. In: Ministério da Saúde, organizador. Gestão municipal de saúde: textos básicos. Brasília: Ministério da Saúde; 2001. p.163-9.

21. Branco MAF. Sistemas de informação em saúde no nível local. Cad Saúde Pública 1996; 12:267-70.

22. Campos LA. Terceirização de serviços públicos. Boletim Jurídico 2006; (190). http://www.bo letimjuridico.com.br/doutrina/texto.asp?id=1470 (acessado em 10/Nov/2008)

23. Alves ON. Terceirização de serviços na administração pública. http://portal2.tcu.gov.br/portal/ pls/portal/docs/769538.PDF (acessado em 05/ Nov/2008).

24. Moreira ML. Sistema de informação de saúde: a epidemiologia e a gestão de serviço. Saúde Soc 1995; 4:43-5.

25. Ferreira VMB, Portela MCP. Avaliação da subnotificação de casos de Aids no Município do Rio de Janeiro com base em dados do sistema de informações hospitalares do Sistema Único de Saúde. Cad Saúde Pública 1999; 15:317-24.

26. Almeida MF, Alencar GP. Informações em saúde: necessidade de introdução de mecanismos de gerenciamento dos sistemas. Inf Epidemiol SUS 2000; 9:241-9.

27. Ministério da Saúde. Sistema de planejamento do SUS: perfil da atividade de planejamento no Sistema Único de Saúde. Brasília: Ministério da Saúde; 2008.

28. Ministério da Saúde. Portaria nº. 91/GM. Diário Oficial da União 2007; 16 jan.

29. Cohn A, Westphal MF, Elias PE. Informação e decisão política em saúde. Rev Saúde Pública 2005; 39:114-21.

Recebido em 22/Mar/2009

Versão final reapresentada em 24/Ago/2009

Aprovado em 10/Nov/2009 